\title{
Patrimonio arquitectónico y urbanístico en Andalucía: Hacia la configuración de un sistema de información territorial
}

\author{
Ramón Pico Valimaña \\ Mariano A. Pérez Humanes
}

Centro de Documentación IAPH

Tras una política cultural que hasta hace unos años ha estado dominada más por la emergencia que por la planificación, actualmente se constata un interés creciente por la elaboración de instrumentos de tutela y divulgación del Patrimonio Histórico que lo pongan en relación con otros con los que se vincula configurando entidades territoriales integradas. Todos los que trabajamos en el Patrimonio somos conscientes de que el conocimiento de los bienes patrimoniales está tendiendo, cada vez, más hacia la búsqueda de las relaciones entre las cosas que al estudio concreto de las cosas mismas, es decir, que las cosas -en este caso los bienes patrimoniales- no son sin sus relaciones. Es por ello que serán las relaciones entre las cosas lo que de alguna manera comiencen a explicarnos lo que cada una es.

Desde este punto de vista, proponemos una aproximación al territorio a través de áreas territoriales homogéneas tanto desde su propia evolución histórica como de las condiciones físicas, socioeconómicas y culturales que las caracterizan a priori. Pero ésto no es más que una estrategia de análisis desde la que poder independizar ámbitos espaciales diferenciados, ya que será en el desarrollo del análisis de las relaciones entre los bienes desde el que irán surgiendo las diversas delimitaciones.

Para abordar un trabajo de esta complejidad, parece necesario recordar cuales son los objetivos básicos en que se fundamenta la ordenación del territorio que se plantea la administración:

a. La cohesión económica y social de toda la población que se integra en la unidad político-administrativa sobre la que se actúa, de forma que se encuentre en similares condiciones de acceso al bienestary a la calidad de vida.

b. El desarrollo equilibrado y sostenible de los recursos. Equilibrado territorialmente y sostenible en la medida en que el aprovechamiento actual de los recursos hipoteque su utilización y aprovechamiento en el futuro.

La referencia al Patrimonio Histórico se recoge en las figuras del planeamiento territorial de una forma clara. Así, en el contenido sustantivo del Plan de
Ordenación del Territorio de Andalucía. (Art. 2 Decreto de Formulación 83/1995 de 28 de marzo) se establecen como criterios de ordenación y objetivos cuatro puntos básicos:

a. Contribuir al desarrollo socioeconómico de la región.

b. Proporcionar al territorio la máxima articulación e integración, interna y con el exterior.

c. Hacer posible la renovación de los recursos naturales y la protección del patrimonio natural e histórico.

d. Contribuir a la elevación y distribución homogénea de la calidad de vida y el bienestar social.

En la actualidad, el desarrollo del modelo territorial en nuestra región se establece a través del Plan de Ordenación del Territorio de Andalucía, los Planes de Ordenación del Territorio Subregionales y los Planes con Incidencia en la Ordenación del Territorio.

En esta última figura, recogida en el Anexo I de la LOTA (Ley de Ordenación del Territorio de Andalucía), cabría encuadrar los planes que ordenen y gestionen la Red de Centros Históricos y el Patrimonio Histórico en general. Ante la limitación jurídica establecida por el contenido del anexo, cabría entonces la formulación de Catálogos de naturaleza urbanística y territorial. Se trata de generar un marco integrado de desarrollo del Patrimonio Histórico y Cultural que forme parte de la estructura global de las políticas de ordenación del territorio en nuestra Comunidad, en un plano de coordinación, mejora de eficacia y coherencia de las actuaciones sectoriales.

Como resultado se propone la inclusión efectiva de las políticas culturales en el instrumento de desarroIlo del planeamiento territorial: el Sistema de Información Territorial. Esta inclusión debe entenderse como una aportación enriquecedora por cuanto ofrece una solución integradora a los problemas producidos por los conflictos de intereses entre las políticas culturales y de ordenación del territorio y urbanismo, contribuyendo al uso y disfrute de los recursos patrimoniales por la sociedad al tiempo que se mejora la calidad de vida de los ciudadanos, en base a la planificación de las intervenciones sobre el territorio y la coordinación de los distintos organismos implicados en el proceso. 
Esta presencia del Patrimonio Histórico en los procesos de definición y gestión de las políticas de ordenación territorial debe contar con una doble vertiente.

La primera es aquella que se vincula al conocimiento del proceso de evolución histórica del territorio, factor necesario para llegar a comprender la dinámica de una estructura territorial en el presente, y útil herramienta de ayuda en la determinación de futuras propuestas de desarrollo.

La segunda vertiente, desde el análisis de los recursos y los riesgos potenciales actuales, es capaz de definir una estrategia válida de desarrollo y protección del Patrimonio Histórico integrado en la dinámica evolutiva de la estructura territorial. En una marcada línea de 'activismo cultural', la presencia de los elementos patrimoniales, y, especialmente de los Centros Históricos, debe contemplarse desde su capacidad de generar desarrollo, mas allá de las reducidas visiones proteccionistas. En este sentido, ya en el desarrollo territorial de las actividades turísticas se reconoce un papel importante a la identificación, valoración y señalamiento de criterios de uso del patrimonio edificado, tanto el urbano como el diseminado en el territorio.

Ya desde una visión mas amplia, la constitución de una Red de Centros Históricos debe contribuir al equilibrio solicitado por la ordenación territorial, mediante la integración en programas de cooperación transfronteriza, con la creación de ejes de desarrollo y cooperación transregional.

Todo ello, lógicamente, sin perder la inevitable referencia a la necesaria protección de recursos culturales dentro de los criterios de desarrollo sostenible, que no sólo debe centrarse en el control de los potenciales riesgos que puedan detectarse como susceptibles de afectar a la integridad física de los Bienes Culturales, sino también en la puesta en valor de los mismos para su uso y disfrute.

\section{EL PATRIMONIO HISTORICO EN LAS RECIENTES EXPERIENCIAS ANDALUZAS DE PLANIFICACIÓN TERRITORIAL}

Si en la formulación de los objetivos y contenidos del planeamiento territorial, precisamente determinada desde las mencionadas Bases para la redacción del Plan de Ordenación del Territorio de Andalucía, encontramos siempre esta importante vinculación al Patrimonio Histórico, la práctica no ha producido aun resultados plenamente satisfactorios.

Analizando los últimos trabajos de planeamiento territorial llevados a cabo en Andalucía, se constata una sólida preocupación por lo patrimonial en sus principios que, a la postre, obtiene un grado de concreción que no responde a las espectativas planteadas.

Así ocurre en el más avanzado de los actuales planes subregionales en desarrollo, el Plan de Ordenación del
Territorio de la Aglomeración Urbana de la Bahía de Cádiz, cuyo documento de Avance (Julio 1997), más allá de asumir las determinaciones legales que afectan a los restos arquitectónicos y yacimientos arqueológicos , tanto urbanos como dispersos, protegidos por la Ley de Patrimonio Histórico de Andalucía, propone la protección activa de algunos de ellos 'a través del tratamiento de sus zonas de protección y del diseño de relaciones con otros espacios de interés metropolitano'. Se trata de elementos como la Zona arqueológica de la sierra de San Cristobal y Doña Blanca, el Puente Zuazo, el Poblado de Sancti Petri o el Castillo del mismo nombre, que gracias a su posición e importancia en el conocimiento de la historia de la Bahía, pasan a integrarse en el Sistema de Espacios Libres Metropolitano. Se trata, desde el planeamiento, de hacer compatibles protección, conocimiento, uso y disfrute de estos espacios, acercándonos a la más moderna visión del patrimonio como recurso. Sin embargo quedan importantes vacíos por cubrir, dado que el trazado de las nuevas infraestructuras metropolitanas y la articulación del Sistema de Espacios Libres no han contado con una cobertura amplia de información arqueológica, arquitectónica y etnológica.

No sabemos si las determinaciones del Plan, sujetas al difícil acuerdo de intereses múltiples, hubieran cambiado de haberse podido contar con dicha información; pero nuestra tarea debe centrarse en ofrecer la mejor base posible para estos análisis, con el objetivo de que las determinaciones del planeamiento estén cada vez más fundamentadas.

Similar planteamiento encontramos en el documento de diagnóstico del Plan de Ordenación del Territorio de la Aglomeración Urbana de Sevilla, donde la preocupación por lo patrimonial se ve eclipsada por la carencia de una información de partida efectiva aportada por la administración de cultura, solicitada en su día por el equipo redactor.

Podríamos decir que la incorporación de un determinado elemento patrimonial al proceso de planificación se viene basando en dos aspectos: por un lado, el grado de protección legal que la administración de cultura asigna al bien; y por otro, la posición relativa de éste respecto al resto de las circunstancias territoriales.

Sin embargo, la inexistencia de un instrumento capaz de incorporarlos desde una clara integración en el territorio está imposibilitando la puesta en juego de los mismos como era de desear. En la coyuntura actual la administración de cultura no dispone, de momento, de herramientas ágiles para el conocimiento y la transmisión de la información patrimonial a nivel territorial; y los complejos y exhaustivos documentos de información y análisis que se elaboran desde los propios planes nunca llegan, en su amplia pero virada visión, a una valoración concreta de los bienes patrimoniales. Es por ello que debemos trabajar no sólo para contar con una base sólida de partida sino para posibilitar el encuentro de las diversas estrategias y posicionamientos.

En el ámbito de la planificación y gestión urbana existe un problema añadido. Se trata en este caso de una si- 
tuación en la que encontramos una gran diversidad, entrando en contraste municipios con planeamiento ampliamente desarrollados frente a otros que han carecido de instrumentos o preocupación por el Patrimonio. En este caso, tenemos que hablar de carencia de un marco normalizador, figura que igualmente puede ser absorbida por un Sistema de Información Territorial capaz de descender, como permiten los SIG, a la escala urbana.

\section{UN PASO ADELANTE}

Estas circunstancias asumidas en la actualidad por todos los que trabajamos en el conocimiento, valoración y tutela del patrimonio, aún no acaban de llevarse a la práctica hasta sus últimas consecuencias, por lo que la dificultad de cualquier trabajo de normalización e informatización de bienes patrimoniales se ve potenciada desde unas inexplicables separaciones entre disciplinas, administraciones y ámbitos ciudadanos que impiden un desarrollo más fluido de los trabajos y hacen de un lugar de encuentro un lugar de conflicto.

Continuando el camino abierto por experiencias ya desarrolladas con éxito de forma incipiente desde el área de Patrimonio Arqueológico, el desarrollo actual de las bases de datos de Patrimonio Arquitectónico y Conjuntos Históricos está dibujando un sólido campo de trabajo tendente a configurar un cuerpo normalizado de información territorial en el futuro.

Se trata entonces de aprovechar el potencial de los Sistemas de Información Geográfica para dar un paso más en el tejido de la red de información patrimonial en la que nos encontramos trabajando. Un paso de gran importancia cualitativa que encaja plenamente con los objetivos generales del SIPHA.

En la actualidad, la base de datos de Patrimonio Arquitectónico cuenta con 3436 registros, pertenecientes esencialmente a bienes legalmente protegidos. Se trata de una herramienta consolidada dentro del Sistema de Información de Patrimonio Histórico cuya estructura ya ha sido abordada en otro número de este Boletín (PH $n^{\circ}$ 17). De su estructura se desgajaría la información de los Conjuntos Históricos, capaz de configurar un núcleo específico en el modelo integrado del SIPHA.

\section{LA BASE DE DATOS DE CONJUNTOS HISTÓRICOS DEL IAPH}

La Base de Datos de Conjuntos Históricos partió de un proyecto que en el seno del Centro de Documentación del IAPH se viene desarrollando desde Febrero del año 1997 con el nombre de "Normalización e Informatización de los Trabajos de Análisis de los Conjuntos Históricos Andaluces". El trabajo realizado desde esa fecha ha consistido en analizar la documentación existente sobre Conjuntos Históricos Andaluces iniciando un proceso de normalización e informatización que ha llegado a configurar lo que hoy es la Base.
Ya en la década de los 90, la preocupación por esta falta de coordinación entre Administraciones y la necesidad de partir de unos documentos lo más completos y homogéneos posibles ha iniciado una cierta normalización de la documentación necesaria para el conocimiento y valoración de estos inmuebles. Al respecto los análisis de diagnóstico tanto de la Consejería de Obras Públicas y Transportes como los de la Consejería de Cultura han ido mejorando la situación, aún cuando estamos en el inicio de un largo camino. En esa misma línea El Programa Regional de Planeamiento en Centros Históricos, aprobado según la Orden de 9 de Mayo de 1994 recoge también "la necesaria coordinación de las Administraciones urbanísticas y culturales en este ámbito", al tiempo que propone programas y líneas de actuación que promulguen la cooperación municipal y el apoyo a la formulación del planeamiento.

Dentro de estas líneas de mejora del conocimiento, valoración y tutela de los Conjuntos Históricos se incardina la Base de Datos de Conjuntos Históricos del IAPH. Como hemos dicho, el primer problema se plantea cuando hay que normalizar una información dispersa y variopinta. Este es un problema que viene de antiguo y con el que todo tipo de investigadores se han topado sin llegar a conseguir un marco mínimamente coherente para sistematizar la documentación existente sobre estos bienes del patrimonio. Surge entonces la necesidad de aclarar que nivel de conocimiento debería recogerse en estas Base de Datos, sobre todo para que sea un instrumento útil a la hora de no sólo consultar la Base como lugar de conocimiento sino para poder valorar un bien de este tipo; es decir, que esta información pudiera servir de base como documentación previa a la cumplimentación de expedientes de inscripción de bienes en el C.G.P.H.A. con unas mínimas garantías.

Desde este punto de vista, el esfuerzo que ahora se realiza con los Conjuntos Históricos debe tener un horizonte más ambicioso en la medida que puede facilitar la cumplimentación de estos futuros expedientes de inscripción, al tiempo que se constituya en un valioso instrumento de difusión y gestión de nuestro patrimonio.

A estas reflexiones que han ido surgiendo simultáneamente al desarrollo del trabajo habría que añadir una mínima reflexión sobre el propio trabajo realizado, sobre sus hallazgos y sus incompletas tareas puestas en marcha.

Como se ha dicho, el trabajo partió del análisis de la documentación existente sobre Conjuntos Históricos de Andalucía con objeto de normalizarla e informatizarla. Esta normalización ha contado con la experiencia desarrollada tanto de la Base de Datos SIBIA (Sistema de Información Base de Inmuebles Andaluces) como de la Base Integrada del SIPHA (Sistema de Información de Patrimonio Histórico de Andalucía), a las que se les está incorporando algunos datos obtenidos de los diversos organismos: Análisis de Diagnóstico de las Consejerías de O. P. T. y la de Cultura, el I.C.A. (Instituto Cartográfico de Andalucía), el I.E.A. (Instituto de Estadística de Andalucía); y de trabajos concretos como el 'Análisis Urbanístico de los Conjuntos Históricos 
de Andalucía. Ciudades Medias y Pequeñas' de la Dirección General de Ordenación del Territorio y Urbanismo y realizado por Juan Manuel Fernández Serdán.

Es necesario destacar también que la singularidad de este conjunto de inmuebles no sólo está suponiendo una compleja adaptación de estas bases de datos sino un reconocimiento de lo más específico y diferencial de la tipología de Conjuntos Históricos, cuestión que nos lleva a contemplar la futura incorporación de este tipo de instrumentos a un sistema integrado de información y gestión.

La normalización no sólo se ha realizado sobre la información alfanúmerica obtenida y contrastada con la terminología del Tesauro de Patrimonio Histórico Andaluz, sino que también ha habido que normalizar los criterios de inclusión de aquellos datos gráficos (planimetría y fotos) que para el conocimiento de este tipo de inmuebles son especialmente necesarios. Todo ello ha necesitado de un buen número de horas dedicadas a la recopilación de esta información en diversos organismos e instituciones así como la puesta en relación de diversos sistemas informáticos con las propias Bases de Datos. Al mismo tiempo se ha ido diseñando los módulos o pantallas de presentación de la base para ir encajando de manera coherente todos los campos. Hasta el momento se han diseñado cuatro módulos los de Datos Básicos, Datos Administrativos, Datos de Protección y de Análisis.

La Base cuenta en la actualidad con 170 Conjuntos Históricos registrados, de los cuales 130 están protegidos (76 Declarados B.I.C. y 54 Incoados B.I.C.), los 40 restantes recogidos en ella responden a Conjuntos Históricos que el Plan General de Bienes Culturales ha considerado de interés y que están iniciando su tramitación para ser protegidos. Los avatares históricos, ya comentados, unidos a la evolución y puesta al dia del concepto de Conjunto Histórico nos depara aún un cierto desequilibrio provincial y comarcal en la muestra presente en la Base. Así encontramos 30 C.H. protegidos en la provincia de Cádiz frente a 5 C.H. en la de Almería.

Probablemente el mayor esfuerzo realizado en los últimos meses, y posiblemente la mayor aportación de este trabajo, haya sido la normalización del campo de descripciones de los Conjuntos Históricos. Con ello hemos querido homogeneizar los criterios de descripción textual y gráfica que aparecían muy difusos en todos los documentos que hemos estudiado. Básicamente se ha partido de la división de la descripción en tres ámbitos o marcos desde los que poder responder a una descripción coherente y homogénea de todos los Conjuntos: Marco Físico, Marco Histórico y Marco Socio-Económico-Cultural. Los tres marcos se están estudiando a su vez en tres ámbitos el territorial, el urbano y el edificatorio, evitando así repeticiones u olvidos a la hora de cumplimentar cada descripción. Este planteamiento se está adecuando a las necesidades de la Base de Datos Integrada del SIPHA y esta siendo motivo de continuada actualización. Sin embargo, ya contamos con un buen número de descripciones donde podemos comprobar los aciertos y

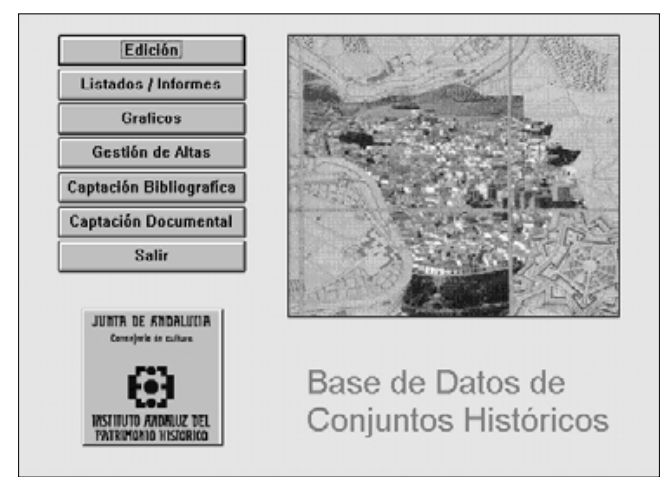

desaciertos de las mismas así como las omisiones más comunes en este tipo de información.

\section{SITUÁNDONOS EN EL TERRITORIO.}

El trabajo de incorporación de estas informaciones a un Sistema de Información Geográfica se ha planteado en tres fases: la primera dirigida a los conjuntos históricos protegidos, con la inclusión de su patrimonio monumental; una segunda que recogería el resto de patrimonio monumental protegido, más allá de los límites administrativos de los conjuntos históricos; para terminar extendiéndonos en una tercera fase, consistente en el registro y diagnóstico definitivo del territorio andaluz.

Desde el IAPH se decide abordar las dos primeras, quedando la última a expensas de una operación de mayor envergadura que necesitaría el apoyo de un grueso trabajo de campo. A lo largo de todo el proceso, resulta incuestionable el marco normalizador de la cartografía, que deberá referirse en cualquier caso a la determinada y disponible de forma homogénea para toda la Comunidad Andaluza por el Instituto de Cartografía de Andalucía.

En la actualidad, este marco es el mosaico ráster georeferenciado a escala I: 10.000. Partiendo de esta base, el trabajo de georeferenciación, descrito en el artículo anterior, se concreta en construir dos coberturas que recojan el patrimonio arquitectónico y una tercera para los conjuntos históricos.

Se establecen así una cobertura de tipo puntual para las localizaciones de los edificios y dos poligonales. La primera recoge las delimitaciones de los entornos de los bienes protegidos y la segunda las delimitaciones de los conjuntos históricos. El partir de una base I: 10.000 limita notablemente las posibilidades a la hora de poder precisar con exactitud dichas delimitaciones e incluso de precisar poligonalmente la extensión de un monumento, pero sin embargo, parece que el esfuerzo necesario para generar estas coberturas está ampliamente compensado con los resultados a obtener. Como primer desarrollo generado con esta herramienta, nos encontramos actualmente preparando, en colaboración con el ICA, un producto de difusión que incorpore en un CD-Rom la información básica de los conjuntos protegidos y sus monumentos. 